\title{
Asymptotic and numerical solutions of three-dimensional boundary-layer flow past a moving wedge
}

\author{
Ramesh B. Kudenatti ${ }^{1}$ (i) | Shashi Prabha Gogate $\mathrm{S}^{1} \mid$ N. M. Bujurke ${ }^{2}$
}

${ }^{1}$ Department of Mathematics, Bangalore University, Central College Campus, Bangalore 560 001, India

${ }^{2}$ Department of Mathematics, Karnatak University, Dharwad 580 003, India

\section{Correspondence}

Ramesh B. Kudenatti, Department of Mathematics, Bangalore University, Central College Campus, Bangalore 560 001, India.

Email: ramesh@bub.ernet.in

Communicated by: T. Monovasilis

MSC Classification: 76D10; 34B05; 65L12
We consider a laminar boundary-layer flow of a viscous and incompressible fluid past a moving wedge in which the wedge is moving either in the direction of the mainstream flow or opposite to it. The mainstream flows outside the boundary layer are approximated by a power of the distance from the leading boundary layer. The variable pressure gradient is imposed on the boundary layer so that the system admits similarity solutions. The model is described using 3-dimensional boundary-layer equations that contains 2 physical parameters: pressure gradient $(\beta)$ and shear-to-strain-rate ratio parameter $(\alpha)$. Two methods are used: a linear asymptotic analysis in the neighborhood of the edge of the boundary layer and the Keller-box numerical method for the full nonlinear system. The results show that the flow field is divided into near-field region (mainly dominated by viscous forces) and far-field region (mainstream flows); the velocity profiles form through an interaction between 2 regions. Also, all simulations show that the subsequent dynamics involving overshoot and undershoot of the solutions for varying parameter characterizing 3-dimensional flows. The pressure gradient (favorable) has a tendency of decreasing the boundary-layer thickness in which the velocity profiles are benign. The wall shear stresses increase unboundedly for increasing $\alpha$ when the wedge is moving in the $x$-direction, while the case is different when it is moving in the $y$-direction. Further, both analysis show that 3 -dimensional boundary-layer solutions exist in the range $-1<\alpha<\infty$. These are some interesting results linked to an important class of boundary-layer flows.

\section{KEYWORDS}

3-dimensional boundary-layer, asymptotics, displacement thickness, Keller box, similarity transformations, wedge flow

\section{1 | INTRODUCTION}

Three-dimensional boundary-layer flow of a viscous and incompressible fluid has attracted many researchers in the recent past because of its relevance to industrial applications such as flow over swept wings, turbine bodies, and flying ball. The 3-dimensional stagnation point flow occurs when a jet of fluid impinges on a rigid body. This is one of the important examples of flow where the 3 velocity components appear. Three-dimensional boundary-layer flow has been developed slowly because we always project the flow phenomena in 2 dimensions. Although, the 2-dimensional boundary-layer flow gives a good deal of simplifications in the Navier-Stokes equations, but it does not reveal actual flow structure. 
The difficulties involved in the analysis of 3-dimensional boundary layers, compared to the 2-dimensional case, are 3 components of velocity and 2 mainstream flows. Since there is an additional flow component, the shear-to-strain-rate or 3-dimensionality parameter $(\alpha)$ appears in the differential equations $\left(\right.$ Banks $\left.^{1}\right)$. Davey ${ }^{2}$ and Davey and Schofield ${ }^{3}$ have analyzed self-similar solutions of the 3-dimensional boundary-layer flows in which the outer mainstream irrotational flows are considered in linear forms $U=U_{\infty} x$ and $V=V_{\infty} y$ (where $U_{\infty}$ and $V_{\infty}$, in our notations, are strain rates and $x$ and $y$ are distances along the surface from the leading edge) in both directions and have reported a wide range of boundary-layer flows including the 2-dimensional case. The solutions exist only in the range $-1 \leq \alpha \leq 0$. However, the flow in the 3-dimensional boundary-layers is mainly dominated by the mainstream flows $U=U_{\infty} x$ and $V=V_{\infty} y$, which are further superposed onto $U(x, y)=U_{\infty} x+V_{\infty} y$ and $V(x, y)=V_{\infty} x+U_{\infty} y$ so that the constraint on $\alpha$ can be removed $\left(W_{e i d m a n}^{4}\right.$ ), and also further extended for the applied magnetic field in the boundary-layer flow (Kudenatti and $\operatorname{Kirsur}^{5}$ ). Note that in these studies, the shear flows form an irrotational flow outside the boundary layer. Further, there is now much evidence, like 2-dimensional case, that the outer mainstream flows can be approximated by a power of distances from the leading boundary-layer edge. Accordingly, the modification of the above mainstream flows in the form $U(x, y)=U_{\infty}(x+y)^{m}$ and $V(x, y)=V_{\infty}(x+y)^{m}$, where $m$ is constant, can provoke significantly a different flow structure and give rational mathematics. We end up yet again with the same shear-to-strain-rate parameter $\alpha=V_{\infty} / U_{\infty}$. The constant $m$ associates with the pressure gradient in the boundary layer since the pressure variation along the normal direction is uniform. For $m>0$, the flow is always accelerated (favorable pressure gradient), while for $m<0$, it is decelerated (an adverse pressure gradient). In addition, with $m=0$, the Blasius boundary layer has a 3-dimensional cousin. In this line of study, Khan et $\mathrm{al}^{6}$ have investigated 3-dimensional boundary-layer flow in a still fluid in which the wedge movement has the above forms in both directions and have shown that for positive values of $m$ (in our notation), the thickness of the boundary layer decreases. The boundary layer forms because of the flow of a viscous fluid over the 2- to 3-dimensional stretching and shrinking porous deforming bodies and the longitudinal fins in rectangular duct (Turkyilmazoglu ${ }^{7}$ ), and the flow model includes both heat transfer and magnetic effect (Turkyilmazoglu ${ }^{8}$ ).

In many practical situations, if the mainstream flow is held constant, the wedge is allowed to move along the streamwise direction, the velocity field forms upstream, and this situation can be found in many practical applications such as manufacturing of polymer sheets and filaments by a continuous extrusion of the polymer from the die to a roller. In this situation, the polymer sheet acts as a moving wedge surface with a nonuniform velocity. And similar applications are also found in metal forming, fiber-processing, magnetic tape production, and so on (Wang ${ }^{9}$; Takhar et al $^{10}$; Tzirtzilakis and Kafousias ${ }^{11}$ ). On the other hand, in a case where the wedge is held constant and mainstream velocity is approximated, in most of the studies, in a linear manner also provides fundamental applications such as in food and paper production and coating of paints on the surface. It is well known that the outer mainstream-velocity variations are approximated in a power-law manner in 3-dimensional flows, which give a broad spectrum of practically important flow configurations including linear case. This type of variations in mainstream flows clearly divides into the accelerated and decelerated flows. Theoretical investigations in the former case, all the solutions in the 3-dimensional boundary layers correspond to entirely forward flow whose thickness of the boundary layer decreases (Dhanak and Duck ${ }^{12}$ ). In the latter case, the flow is massively displaced away from the surface and sometimes is partly reversed.

However, for the mainstream flows defined previously, we follow a slightly different procedure to that first used in Khan et al, ${ }^{6}$ Wang, ${ }^{9}$ Liu et al,,${ }^{13}$ Hayat et al,${ }^{14}$ and Muhammad et al.${ }^{15}$ We introduce a new set of similarity transformations in the governing equations so that we get a shear-to-strain-rate ratio or 3-dimensionality parameter (which is absent in their analyses). Thus, we will focus our study on more global nature of the flow field, which would involve inevitably a full discussion of the boundary-layer flow with respect to the shear-to-strain-rate ratio parameter. We will also focus on the effects of pressure gradient on 3-dimensional boundary layer flows over moving wedge possessing the horizontal velocity behaviors.

The remainder of this paper is organized as follows. In Section 2, we present the problem formulation of 3-dimensional flow over a moving wedge surface. Using similarity transformations, the governing equations have been transformed into a coupled third-order ordinary differential equations, which are nonlinear in nature. In Section 3, we give an asymptotic solution of the 3-dimensional boundary-layer equations in the limit of large $\eta$ (far-field behavior). In this case, we get the coupled linear ordinary differential equations, which are solved through the linear algebraic approach in terms of the confluent hypergeometric functions. For the full nonlinear system, the Keller-box numerical scheme (ie, implicit finite-difference scheme) is used. The procedure has already been used in several complicated problems and also in most of the boundary-layer problems. The procedure of the method and convergence criterion relevant to the problem is given in Section 4. In Section 5, we present and discuss results of the velocity profiles, wall shear stresses, and displacement thicknesses for various parameters in detail. 


\section{2 | FLOW THEORY}

We consider the 3-dimensional laminar boundary-layer flow over a wedge surface in a viscous and incompressible fluid. The Cartesian coordinates are used in which the $x$ - and $y$-axes are measured along the streamwise directions of the mainstream flows and $z$-axis is normal to the flow. The fluid occupies the half-space $z>0$. We consider the mainstream flows with velocities $U(x, y)$ and $V(x, y)$ in both $x$ - and $y$-directions. When the mainstreams flow with large Reynolds number over a wedge, which is moving either in the same direction or opposite, thin boundary layers form on the surface and grow gradually downstream. In these boundary layers, the viscosity effects are significant, and any velocity variations in the normal direction ( $z$-axis) are predominantly more than the streamwise directions ( $x$ - and $y$-axes), ie, $\partial_{z}(u, v) \gg\left(\partial_{x}, \partial_{y}\right)(u, v)$. Also if $\delta$ is the thickness of the boundary layer, then $\delta \ll L$, where $L$ is the length of wedge surface. In addition, we define the following independent dimensionless variables: $x^{*}=\frac{x}{L}, y^{*}=\frac{y}{L}, z^{*}=\frac{z}{\delta}, \mathbf{q}^{*}=\frac{\mathbf{q}}{\mathbf{U}}, p^{*}=\frac{p}{P_{\infty}}$, where $\mathbf{U}, P_{\infty}$ are certain reference quantities, $\mathbf{q}$ is the velocity vector, and $p$ is the pressure (Schlichting and Gersten ${ }^{16}$ ). Since the pressure variation is uniform throughout the flow field and $\frac{\partial p}{\partial z}=0$, outside the boundary layer, we have $u=U$, and $v=V$, which lead to $-\frac{1}{\rho} \frac{\partial p}{\partial x}=U \frac{\partial U}{\partial x}+V \frac{\partial U}{\partial y},-\frac{1}{\rho} \frac{\partial p}{\partial y}=U \frac{\partial V}{\partial x}+V \frac{\partial V}{\partial y}$ (from the Bernoulli theory). Introducing these parameters and above boundary-layer approximations into the following steady and incompressible Navier-Stokes and the continuity equations

$$
\begin{gathered}
(\mathbf{q} \cdot \nabla) \mathbf{q}=-\frac{1}{\rho} \nabla p+\nu \nabla^{2} \mathbf{q}, \\
\nabla \cdot \mathbf{q}=0,
\end{gathered}
$$

where $\rho$ and $v$ are the density and the kinematic viscosity of the fluid, we get the following equations:

$$
\begin{gathered}
u \frac{\partial u}{\partial x}+v \frac{\partial u}{\partial y}+w \frac{\partial u}{\partial z}=U \frac{\partial U}{\partial x}+V \frac{\partial U}{\partial y}+v \frac{\partial^{2} u}{\partial z^{2}} \\
u \frac{\partial v}{\partial x}+v \frac{\partial v}{\partial y}+w \frac{\partial v}{\partial z}=U \frac{\partial V}{\partial x}+V \frac{\partial V}{\partial y}+v \frac{\partial^{2} v}{\partial z^{2}} \\
\frac{\partial u}{\partial x}+\frac{\partial v}{\partial y}+\frac{\partial w}{\partial z}=0
\end{gathered}
$$

where $u, v$, and $w$ are velocity components in $x-, y$-, and $z$-directions. The physical boundary conditions for the model are

$$
\begin{aligned}
& \text { at } z=0: \quad u=U_{w}(x, y), \quad v=V_{w}(x, y), \quad w=0, \quad \text { and } \\
& \text { as } z \rightarrow \infty: \quad u \rightarrow U(x, y), \quad v \rightarrow V(x, y),
\end{aligned}
$$

where $U_{w}(x, y)$ and $V_{w}(x, y)$ are the wedge surface velocities in $x$ - and $y$-directions, respectively. These are similar to nonlinear stretching rate of the surface, which are stretched in both lateral directions. It is well-known that the thickness of the boundary layers must increase with distances $x$ and $y$ from the leading edge of the boundary layer. It is therefore expected that both velocities $U_{w}(x, y)$ and $V_{w}(x, y)$ are approximated by the forms $U_{w}(x, y)=U_{0}(x+y)^{m}, V_{w}(x, y)=V_{0}(x+y)^{m}$, where $U_{0}$ and $V_{0}$ are constants. From the boundary conditions, it is evident that all the solutions smoothly approach the mainstream flows asymptotically from the surface velocity. This asymptotic nature of governing Equations 3 to 4 shall be explored shortly. The outer mainstreams are also approximated by a power of distance from the leading boundary layer, ie, $U=U(x, y)=U_{\infty}(x+y)^{m}$ and $V=V(x, y)=V_{\infty}(x+y)^{m}$, where $U_{\infty}, V_{\infty}$, and $m$ have been defined in Section 1 . Note that, the mainstream flows are 3-dimensional, a single streamfunction does not exist. For this reason, we choose 2 streamfunctions $\phi_{1}(x, y, z)$ and $\phi_{2}(x, y, z)$ exactly similar to that of the 2-dimensional flows, in the form:

$$
u=\frac{\partial \phi_{1}}{\partial z}, \quad v=\frac{\partial \phi_{2}}{\partial z}, \quad w=-\left(\frac{\partial \phi_{1}}{\partial x}+\frac{\partial \phi_{2}}{\partial y}\right),
$$

which satisfy the continuity equation automatically and then defining the following similarity transformations:

$$
\phi_{1}=\sqrt{\frac{2 v(x+y)}{(1+m) U}} U f(\eta), \quad \phi_{2}=\sqrt{\frac{2 v(x+y)}{(1+m) U}} \operatorname{Vg}(\eta), \quad \eta=\sqrt{\frac{(1+m) U}{2 v(x+y)}} z
$$


for the above streamfunctions given in (5). Plugging the ansatz defined in (5) and (6) in the momentum boundary-layer equations (3), we get the coupled nonlinear ordinary differential equations as

$$
\begin{aligned}
& f^{\prime \prime \prime}+(f+\alpha g) f^{\prime \prime}+\beta\left(1-f^{\prime 2}\right)+\alpha \beta\left(1-f^{\prime} g^{\prime}\right)=0, \\
& g^{\prime \prime \prime}+(f+\alpha g) g^{\prime \prime}+\alpha \beta\left(1-g^{2}\right)+\beta\left(1-f^{\prime} g^{\prime}\right)=0,
\end{aligned}
$$

and the boundary conditions (4) accordingly become

$$
f(0)=g(0)=0, \quad f^{\prime}(0)=\lambda_{1}, \quad g^{\prime}(0)=\lambda_{2}, \quad f^{\prime}(+\infty) \rightarrow 1, \quad f^{\prime}(+\infty) \rightarrow 1,
$$

where $f=f(\eta)$ and $g=g(\eta)$ are nondimensional streamfunctions, $\eta$ is a similarity variable, and a prime denotes differentiation with respect to $\eta$. The parameter $\alpha=\frac{V_{\infty}}{U_{\infty}}$ is the shear-to-strain-rate ratio parameter or 3-dimensionality. For $\alpha=0$, the system reduces to 2-dimensional classical boundary-layer flows. The new parameter $\beta=\frac{2 m}{1+m}$ is a measure of the pressure gradient, which is associated with mainstream forcing on the boundary layer, $\beta>0$ represents a favorable pressure gradient and $\beta<0$ an adverse pressure gradient, and $\beta=0$ denotes the flow over a flat plate at zero incidence. When $\alpha=0$ and $\beta=1$, the system converted to Hiemenz flow. Further, $\lambda_{1}=\frac{U_{0}}{U_{\infty}}, \lambda_{2}=\frac{V_{0}}{V_{\infty}}$ are the ratios of freestream velocity to boundary velocity. Note that $\lambda_{1}, \lambda_{2}>0$ and $\lambda_{1}, \lambda_{2}<0$ correspond to moving wedge surface in the same and in the opposite directions to that of freestream velocity, whereas $\lambda_{1}=0=\lambda_{2}$ is the case for flow over a fixed wedge. The other cases of $\lambda_{1}$ and $\lambda_{2}$ shall be discussed later. For the case of moving surface in a still fluid, Tzirtzilakis and Kafoussias ${ }^{11}$ have explored for equal speeds of stretching in lateral directions $\left(\lambda_{1}=\lambda_{2}=1\right.$ in our notations).

The system (7)-(8) describes 3-dimensional boundary-layer flows over a moving surface and contains 4 important physical parameters $\left(\alpha, \beta, \lambda_{1}\right.$, and $\left.\lambda_{2}\right)$. Flow is induced because of the streamwise pressure gradient and by the motion of the wedge in both directions. In other words, the main fluid dynamics is analyzed with respect to both moving wedge and pressure gradient. Since the system is governed by coupled nonlinear ordinary differential equations, any analytical solutions are usually not possible, we resort to numerical solution using the finite difference-based Keller-box method. However, before solving the nonlinear system, we adopt quite different approach to draw some analytical conclusions on the system. In the following section, we give the asymptotic solution of the governing system, in particular far-field behavior of the system, ie, for $\eta \gg 1$.

\section{3 | ASYMPTOTICS FOR FAR-FIELD BEHAVIOR}

The velocity curves in the boundary layer approach their outer mainstream flows smoothly. Upon analysis of the nature of the derivative boundary conditions at infinity, ie, $f^{\prime}(\eta) \rightarrow 1, g^{\prime}(\eta) \rightarrow 1$ as $\eta \rightarrow \infty$, it is appropriate to consider $f(\eta)$ and $g(\eta)$ in the forms

$$
f(\eta) \sim \eta+V c_{1}+\int \mathcal{F}(\eta) d \eta, \quad g(\eta) \sim \eta+V c_{2}+\int \mathcal{G}(\eta) d \eta
$$

where $V c_{1}$ and $V c_{2}$ are integration constants and $\mathcal{F}(\eta)$ and $\mathcal{G}(\eta)$ and their derivatives are assumed small. Note that both $\mathcal{F}(\eta) \rightarrow 0$ and $\mathcal{G}(\eta) \rightarrow 0$ as $\eta \rightarrow \infty$. The recent interesting work of Kudenatti et al ${ }^{17}$ tackles an equally valid and rather related problem but for 2-dimensional boundary-layer flow over a wedge and obtained the solution in the form of confluent hypergeometric functions. Both $V c_{1}=0=V c_{2}$ from the boundary conditions (8). Substituting (9) into (7) and upon linearizing the resulting equations, we get linear coupled ordinary differential equations:

$$
\begin{aligned}
& \mathcal{F}^{\prime \prime}(\eta)+(\alpha+1) \eta \mathcal{F}^{\prime}(\eta)-(2+\alpha) \beta \mathcal{F}(\eta)-\alpha \beta \mathcal{G}(\eta)=0, \\
& \mathcal{G}^{\prime \prime}(\eta)+(\alpha+1) \eta \mathcal{G}^{\prime}(\eta)-\beta \mathcal{F}(\eta)-(2 \alpha+1) \beta \mathcal{G}(\eta)=0
\end{aligned}
$$

with the boundary conditions

$$
\mathcal{F}(0)=\lambda_{1}-1, \quad \mathcal{G}(0)=\lambda_{2}-1, \quad \mathcal{F}(+\infty)=0, \quad \mathcal{G}(+\infty)=0
$$


Equations 10 and 11 give a good deal of a mathematical simplification. For $\beta=0$, the exact solutions of (10) and (11) are given by

$$
\mathcal{F}(\eta)=\left(\lambda_{1}-1\right) \operatorname{erfc}(\hat{\alpha} \eta), \quad \mathcal{G}(\eta)=\left(\lambda_{2}-1\right) \operatorname{erfc}(\hat{\alpha} \eta),
$$

where $\operatorname{erfc}(\cdot)$ is the complementary error function and $\hat{\alpha}=\sqrt{2 /(1+\alpha)}, \alpha \neq-1$. For $\beta \neq 0$, the above system (10) can be written for convenience as

$$
\begin{aligned}
& \mathcal{F}^{\prime \prime}(\eta)+\hat{a} \eta \mathcal{F}^{\prime}(\eta)+a \mathcal{F}(\eta)+b \mathcal{G}(\eta)=0, \\
& \mathcal{G}^{\prime \prime}(\eta)+\hat{a} \eta \mathcal{G}^{\prime}(\eta)+c \mathcal{F}(\eta)+d \mathcal{G}(\eta)=0,
\end{aligned}
$$

where $\hat{a}=(1+\alpha), a=-(\alpha+2) \beta, b=-\alpha \beta, c=-\beta$, and $d=-(1+2 \alpha) \beta$, although the system (13) is linear second-order ordinary differential equation but cannot be solved exactly in the present form because of coupled equations. To obtain the solutions for (13), we need to decouple the equations. Writing the horizontal velocity profiles $\mathcal{F}(\eta)$ and $\mathcal{G}(\eta)$ in $(13)$ in the matrix form

$$
\left(\begin{array}{l}
\mathcal{F}^{\prime \prime} \\
\mathcal{G}^{\prime \prime}
\end{array}\right)(\eta)+\hat{a} \eta\left(\begin{array}{ll}
1 & 0 \\
0 & 1
\end{array}\right)\left(\begin{array}{l}
\mathcal{F}^{\prime} \\
\mathcal{G}^{\prime}
\end{array}\right)(\eta)+\left(\begin{array}{ll}
a & b \\
c & d
\end{array}\right)\left(\begin{array}{l}
\mathcal{F} \\
\mathcal{G}
\end{array}\right)(\eta)=\left(\begin{array}{l}
0 \\
0
\end{array}\right)
$$

shows that the problem may be solved by diagonalizing (14). Further, defining

$$
S(\eta)=\left(\begin{array}{l}
\mathcal{F} \\
\mathcal{G}
\end{array}\right)(\eta), \quad I=\left(\begin{array}{ll}
1 & 0 \\
0 & 1
\end{array}\right), \quad A=\left(\begin{array}{ll}
a & b \\
c & d
\end{array}\right)
$$

the above system (14) reduces

$$
S^{\prime \prime}(\eta)+I \hat{a} \eta S^{\prime}(\eta)+A S(\eta)=0 .
$$

Note that the matrix $A$ entirely depends on both physical parameters $\alpha$ and $\beta(\neq 0)$. The matrix $A$ is diagonalizable if it has distinct eigenvalues. Using standard results of computational linear algebra, the diagonalizable matrix $A$ may be written as

$$
A=P^{-1} D P \text {. }
$$

Here, both $P$ and $D$ are given by

$$
P=\left(\begin{array}{ll}
a_{11} & a_{12} \\
a_{21} & a_{22}
\end{array}\right), \quad D=\left(\begin{array}{cc}
\Delta_{1} & 0 \\
0 & \Delta_{2}
\end{array}\right)
$$

where both columns of $P$ are the eigenvectors

$$
a_{11}=-\frac{-a+d+\sqrt{a^{2}+4 b c-2 a d+d^{2}}}{2 c}, a_{12}=-\frac{-a+d-\sqrt{a^{2}+4 b c-2 a d+d^{2}}}{2 c}, \text { and } a_{21}=a_{22}=1,
$$

corresponding to the eigenvalues

$$
\Delta_{1}=\frac{a+d+\sqrt{(a+d)^{2}-4(a d-b c)}}{2}, \quad \Delta_{2}=\frac{a+d-\sqrt{(a+d)^{2}-4(a d-b c)}}{2},
$$

determined from $|A-I \Delta|=0$. These eigenvalues $\Delta_{1}$ and $\Delta_{2}$ dictate the external mainstream velocities to flow only in $x$ - and $y$-directions separately. This means that the mainstream flows now become $U(x)=U_{\infty} x^{m}, V(y)=V_{\infty} y^{m}$ with variables having same definitions. Defining $P S(\eta)=T(\eta)$, where $T(\eta)=\left(T_{1}, T_{2}\right)^{t}(\eta)$. Using (17), the system (16) may be written as

$$
T^{\prime \prime}(\eta)+\hat{a} \eta T^{\prime}(\eta)+D T(\eta)=0
$$

where

$$
\left(\begin{array}{l}
T_{1}^{\prime \prime} \\
T_{2}^{\prime \prime}
\end{array}\right)(\eta)+\hat{a} \eta\left(\begin{array}{ll}
1 & 0 \\
0 & 1
\end{array}\right)\left(\begin{array}{l}
T_{1}^{\prime} \\
T_{2}^{\prime}
\end{array}\right)(\eta)+\left(\begin{array}{cc}
\Delta_{1} & 0 \\
0 & \Delta_{2}
\end{array}\right)\left(\begin{array}{l}
T_{1} \\
T_{2}
\end{array}\right)(\eta)=\left(\begin{array}{l}
0 \\
0
\end{array}\right)
$$


is the decoupled system of equations, $\mathrm{cf}(14)$ and (22). The complete solution of the system (21) is given by

$$
T_{1,2}(\eta)=A_{1,2}\left[\mathcal{M}\left(1-K_{1,2}, \frac{1}{2},-\frac{\hat{a}}{2} \eta^{2}\right)-\frac{2 \Gamma\left(K_{1,2}\right)}{\Gamma\left(K_{1,2}-\frac{1}{2}\right)} \sqrt{\frac{\hat{a}}{2}} \eta \mathcal{M}\left(\frac{3}{2}-K_{1,2}, \frac{3}{2},-\frac{\hat{a}}{2} \eta^{2}\right)\right],
$$

where $\mathcal{M}(\cdot, \cdot, \eta)$ is the confluent hypergeometric function of first kind (Abramowitz and Stegum ${ }^{18} ;$ Andrews ${ }^{19}$ ) and $A_{1,2}=$ $\lambda_{1,2}-1, \quad K_{1,2}=1-\frac{\Delta_{1,2}}{2 \hat{a}}, \Gamma$ is the Gamma function. Note that the first and second suffixes in each variable denote the first and second component solutions of (21) (like $T_{1}=A_{1}, \lambda_{1}$, and $K_{1}$ ). Finally, since $P S(\eta)=T(\eta)$ or $S(\eta)=P^{-1} T(\eta)$, both velocity profiles $\mathcal{F}(\eta)$ and $\mathcal{G}(\eta)$ in (14) are given by

$$
\left(\begin{array}{l}
\mathcal{F} \\
\mathcal{G}
\end{array}\right)(\eta)=\frac{1}{|P|}\left(\begin{array}{cc}
a_{22} & -a_{12} \\
-a_{21} & a_{11}
\end{array}\right)\left(\begin{array}{l}
T_{1} \\
T_{2}
\end{array}\right)(\eta)
$$

where $T_{1}$ and $T_{2}$ are obtained from (23). The velocity profiles are given by

$$
f^{\prime}(\eta)=1+\mathcal{F}(\eta), \quad g^{\prime}(\eta)=1+\mathcal{G}(\eta) .
$$

The velocity profiles in a 3-dimensional boundary layer with prescribed mainstream flows are obtained by (25). For these asymptotic solutions to be valid in the neighborhood of edge of the boundary layer, both $\mathcal{F}(\eta) \rightarrow 0$ and $\mathcal{G}(\eta) \rightarrow 0$ as $\eta \rightarrow \infty$. It is also of interest that the wall shear stresses and the displacement thicknesses

$$
\begin{gathered}
\tau=\mu\left[\frac{\partial u}{\partial z} i+\frac{\partial v}{\partial z} j\right]_{z=0}=\mu\left(\frac{m+1}{2 v(x+y)}\right)^{\frac{1}{2}} U^{\frac{3}{2}}\left[\mathcal{F}^{\prime}(0) i+\alpha \mathcal{G}^{\prime}(0) j\right] \\
\quad \text { and } \quad \delta_{x 0}=\int_{0}^{\infty}-\mathcal{F}(\eta) d \eta, \quad \delta_{y 0}=\int_{0}^{\infty}-\mathcal{G}(\eta) d \eta
\end{gathered}
$$

where $i$ and $j$ are unit vectors directed along the $x$ - and $y$-axes and the required quantities are obtained from (25), can be obtained for various physical parameters.

To understand any flow variation near the wedge surface, it is instructive to consider the full nonlinear system with the same pressure gradient and 3-dimensionality parameter. These aspects are addressed in the below section.

\section{4 | NUMERICAL SOLUTION}

The boundary value problems posed by (7) subjected to boundary conditions (8) are solved numerically. Since the system is nonlinear and coupled, the Keller-box method, which is efficient and second-order convergence, is used to solve the full nonlinear system (7) and (8). Various details of the method can be found in Cebeci and Bradshaw ${ }^{20}$ and Keller. ${ }^{21}$ This method is based on the implicit finite-difference and involves various steps. The system first needs to be converted into a system of first-order equations by introducing some additional unknown functions. This first-order system is discretized using central finite-difference scheme, which produces a nonlinear system of algebraic equations. We linearize them using Newton linearization technique. Write the linearized system of equations in matrix vector form and the Lower-Upper decomposition method is used for its solution and update the correction at each iteration until we obtain the convergent solution within a tolerance limit of $10^{-8}$.

To describe this method, the system (7) with boundary conditions (8) is rewritten in the form of system of first-order ordinary differential equations, which are given by

$$
\begin{aligned}
& \frac{d f}{d \eta}=E, \quad \frac{d E}{d \eta}=F, \quad \frac{d F}{d \eta}=-\left(F(f+\alpha g)+\beta\left(1-E^{2}\right)+\beta \alpha(1-E G)\right), \\
& \frac{d g}{d \eta}=G, \quad \frac{d G}{d \eta}=H, \quad \frac{d H}{d \eta}=-\left(H(f+\alpha g)+\alpha \beta\left(1-G^{2}\right)+\beta(1-E G)\right),
\end{aligned}
$$

and the respective boundary condition are given by 


$$
\begin{array}{rcr}
f=g=E=G=0 \quad \text { when } & \eta=0, \\
E \rightarrow 1, G \rightarrow 1 & \text { as } \quad \eta \rightarrow \infty .
\end{array}
$$

Using suitable finite difference approximations, namely

$$
f=\frac{f_{i}+f_{i-1}}{2}, \frac{d f}{d \eta}=\frac{f_{i}-f_{i-1}}{\eta_{i}}, \text { etc, }
$$

where $\eta_{i}$ is the step length in $\eta$-direction. Using Equation (29) in (27) and simplifying, we get

$$
\begin{gathered}
f_{i}-f_{i-1}=\frac{\eta_{i}}{2}\left(E_{i}+E_{i-1}\right), \\
E_{i}-E_{i-1}=\frac{\eta_{i}}{2}\left(F_{i}+F_{i-1}\right), \\
g_{i}-g_{i-1}=\frac{\eta_{i}}{2}\left(G_{i}+G_{i-1}\right), \\
G_{i}-G_{i-1}=\frac{\eta_{i}}{2}\left(H_{i}+H_{i-1}\right), \\
F_{i}-F_{i-1}=-\frac{\eta_{i}}{4}\left(\left(F_{i}+F_{i-1}\right)\left(f_{i}+f_{i-1}+\alpha\left(g_{i}+g_{i-1}\right)\right)+\beta\left(4-\left(E_{i}+E_{i-1}\right)^{2}\right)+\beta \alpha\left(4-\left(E_{i}+E_{i-1}\right)\left(G_{i}+G_{i-1}\right)\right)\right), \\
H_{i}-H_{i-1}=-\frac{\eta_{i}}{4}\left(\left(H_{i}+H_{i-1}\right)\left(f_{i}+f_{i-1}+\alpha\left(g_{i}+g_{i-1}\right)\right)+\alpha \beta\left(4-\left(G_{i}+G_{i-1}\right)^{2}\right)+\beta\left(4-\left(E_{i}+E_{i-1}\right)\left(G_{i}+G_{i-1}\right)\right)\right) .
\end{gathered}
$$

The above system of equations exhibits nonlinear algebraic equations, which are difficult to solve. Therefore, we linearize them by introducing

$$
\mathbf{a}^{(k+1)}=\mathbf{a}^{(k)}+\delta \mathbf{a}^{(k)},
$$

where $\mathbf{a}=[f, E, F, g, G, H]^{T}$. Substituting (31) in (30) and any products of $\delta \mathbf{a}$ can be neglected, we get system of linear algebraic equations in $\delta \mathbf{a}^{(k)}$ as

$$
\begin{gathered}
\delta f_{i}+\delta f_{i-1}-d \delta E_{i}-d \delta E_{i-1}=f_{i-1}-f_{i}+d E_{i}+d E_{i-1}, \\
\delta E_{i}+\delta E_{i-1}-d \delta F_{i}-d \delta F_{i-1}=E_{i-1}-E_{i}+d F_{i}+d F_{i-1}, \\
\delta g_{i}+\delta g_{i-1}-d \delta G_{i}-d \delta G_{i-1}=g_{i-1}-g_{i}+d G_{i}+d G_{i-1}, \\
\delta G_{i}+\delta G_{i-1}-d \delta H_{i}-d \delta H_{i-1}=G_{i-1}-G_{i}+d H_{i}+d H_{i-1}, \\
a_{1} \delta F_{i}+a_{2} \delta F_{i-1}+a_{3}\left(\delta f_{i}+\delta f_{i-1}\right)+a_{4}\left(\delta g_{i}+\delta g_{i-1}\right)+a_{5}\left(\delta E_{i}+\delta E_{i-1}\right)+a_{6}\left(\delta G_{i}+\delta G_{i-1}\right)=-2 d \beta(1+\alpha) \\
+F_{i-1}+F_{i}-\frac{d}{2}\left(F_{i}+F_{i-1}\right)\left(f_{i}+f_{i-1}\right)-\frac{d \alpha}{2}\left(F_{i}+F_{i-1}\right)\left(g_{i}+g_{i-1}\right) \\
+\frac{d \beta}{2}\left(E_{i}+E_{i-1}\right)^{2}+\frac{d \alpha \beta}{2}\left(E_{i}+E_{i-1}\right)\left(G_{i}+G_{i-1}\right), \\
b_{1} \delta H_{i}+H \delta g 2_{i-1}+b_{3}\left(\delta f_{i}+\delta f_{i-1}\right)+b_{4}\left(\delta g_{i}+\delta g_{i-1}\right)+b_{5}\left(\delta E_{i}+\delta E_{i-1}\right)+b_{6}\left(\delta G_{i}+\delta G_{i-1}\right)=-2 d \beta \alpha \\
+H_{i-1}+H_{i}-\frac{d}{2}\left(H_{i}+H_{i-1}\right)\left(f_{i}+f_{i-1}\right) \\
-\frac{d \alpha \beta}{2}\left(H_{i}+H_{i-1}\right)\left(g_{i}+g_{i-1}\right)+\frac{d \beta \alpha}{2}\left(G_{i}+G_{i-1}\right)^{2}+\frac{d \beta}{2}\left(E_{i}+E_{i-1}\right)\left(G_{i}+G_{i-1}\right)
\end{gathered}
$$

with 


$$
\begin{aligned}
& a_{1}=1+\frac{d}{2}\left(f_{i}+f_{i-1}\right)+\frac{\alpha d}{2}\left(g_{i}+g_{i-1}\right), \quad a_{2}=-1+\frac{d}{2}\left(f_{i}+f_{i-1}\right)+\frac{\alpha d}{2}\left(g_{i}+g_{i-1}\right), \\
& a_{3}=\frac{d}{2}\left(F_{i}+F_{i-1}\right), \quad a_{4}=\frac{\alpha d}{2}\left(F_{i}+F_{i-1}\right), \quad a_{5}=-d \beta\left(E_{i}+E_{i-1}\right)+\frac{d \beta \alpha}{2}\left(G_{i}+G_{i-1}\right), \quad a_{6}=-\frac{d \beta \alpha}{2}\left(E_{i}+E_{i-1}\right) \\
& b_{1}=1+\frac{d}{2}\left(f_{i}+f_{i-1}\right)+\frac{\alpha d}{2}\left(g_{i}+g_{i-1}\right), \quad b_{2}=-1+\frac{d}{2}\left(f_{i}+f_{i-1}\right)+\frac{\alpha d}{2}\left(g_{i}+g_{i-1}\right), \\
& b_{3}=\frac{d}{2}\left(H_{i}+H_{i-1}\right), \quad b_{4}=\frac{\alpha d}{2}\left(H_{i}+H_{i-1}\right), \quad b_{5}=-\frac{d \beta \alpha}{2}\left(G_{i}+G_{i-1}\right), \\
& b_{6}=-d \alpha \beta\left(G_{i}+G_{i-1}\right)+\frac{d \beta}{2}\left(E_{i}+E_{i-1}\right), \quad d=\frac{\eta_{i}}{2} .
\end{aligned}
$$

The above system of Equations 32 can conveniently be put in the matrix form:

$$
\mathbf{A D}=\mathbf{R},
$$

where

$$
\mathbf{A}=\left[\begin{array}{ccccc}
{\left[A_{1}\right]} & {\left[C_{1}\right]} & 0 & \ldots & \ldots \\
{\left[B_{2}\right]} & {\left[A_{2}\right]} & {\left[C_{2}\right]} & 0 & \ldots \\
0 & {\left[B_{3}\right]} & {\left[A_{3}\right]} & {\left[C_{3}\right]} & \ldots \\
\ldots & \ldots & \ldots & \ldots & \ldots \\
0 & \ldots & {\left[B_{i-1}\right]} & {\left[A_{i-1}\right]} & {\left[C_{i-1}\right]} \\
0 & \ldots & \ldots & {\left[B_{i}\right]} & {\left[A_{i}\right]}
\end{array}\right]
$$

where $\mathbf{A}$ is a block-tridiagonal structure and each element of $\mathbf{A}$ is again a matrix of order six, $\mathbf{D}$ is column matrix $[\delta f, \delta E, \delta F, \delta g, \delta G, \delta H]^{T}$ as unknown entries, and $\mathbf{R}$ is the right hand of the (32). The entries of matrix $\mathbf{A}$ are given by

$$
\begin{aligned}
& A_{1}=\left[\begin{array}{cccccc}
0 & 0 & 1 & 0 & 0 & 0 \\
-d & 0 & 0 & -d & 0 & 0 \\
0 & 0 & 0 & 0 & 1 & 0 \\
0 & -d & 0 & 0 & 0 & -d \\
a_{2} & 0 & a_{3} & a_{1} & a_{4} & 0 \\
0 & b_{2} & b_{3} & 0 & b_{4} & b_{1}
\end{array}\right], \quad A_{j}=\left[\begin{array}{cccccc}
-d & 0 & 1 & 0 & 0 & 0 \\
-1 & 0 & 0 & -d & 0 & 0 \\
0 & -d & 0 & 0 & 1 & 0 \\
0 & -1 & 0 & 0 & 0 & 0 \\
a_{5} & a_{6} & a_{3} & a_{1} & a_{4} & 0 \\
b_{6} & b_{5} & b_{3} & 0 & b_{4} & b_{1}
\end{array}\right], \\
& B_{j}=\left[\begin{array}{cccccc}
0 & 0 & -1 & 0 & 0 & 0 \\
0 & 0 & 0 & -d & 0 & 0 \\
0 & 0 & 0 & 0 & -1 & 0 \\
0 & 0 & 0 & 0 & 0 & -d \\
0 & 0 & a_{3} & a_{2} & a_{4} & 0 \\
0 & 0 & b_{3} & 0 & b_{4} & b_{2}
\end{array}\right], \quad C_{j-1}=\left[\begin{array}{cccccc}
-d & 0 & 0 & 0 & 0 & 0 \\
1 & 0 & 0 & 0 & 0 & 0 \\
0 & -d & 0 & 0 & 0 & 0 \\
0 & 1 & 0 & 0 & 0 & 0 \\
a_{5} & a_{6} & 0 & 0 & 0 & 0 \\
b_{6} & b_{5} & 0 & 0 & 0 & 0
\end{array}\right],
\end{aligned}
$$

where $j=2,3,4, \ldots N$. Using the LU decomposition method, the tridiagonal system (33) can be solved for the unknown D and update (31) at each correction until the convergence.

Since physical domain in this problem is unbounded, but the computational domain has to be finite, we apply the far-field boundary conditions for the pseudo-similarity variable $\eta$ at a finite value, which is sufficient to achieve asymptotically the far-field boundary conditions, for all values of the pertinent parameters considered. Our computational code adopts a variable step size and different flow domains to achieve the values of wall shear stresses $f^{\prime \prime}(0)$ and $g^{\prime \prime}(0)$. Once the solutions are obtained, the 2-dimensional boundary-layer displacement thicknesses $\delta_{x}$ and $\delta_{y}$ in $x$ - and $y$-directions are calculated from the relations

$$
\tau=\mu\left[\frac{\partial u}{\partial z} i+\frac{\partial v}{\partial z} j\right]_{z=0}=\mu\left(\frac{m+1}{2 v(x+y)}\right)^{\frac{1}{2}} U^{\frac{3}{2}}\left[f^{\prime \prime}(0) i+\alpha g^{\prime \prime}(0) j\right]
$$

and

$$
\delta_{x}=\int_{0}^{\infty}\left(1-f^{\prime}(\eta)\right) d \eta, \quad \delta_{y}=\int_{0}^{\infty}\left(1-g^{\prime}(\eta)\right) d \eta
$$

numerically. Some discussion of the Keller-box numerical results for 3-dimensional boundary-layer flows for a wide range of parameters are given in the next section. 


\section{5 | RESULTS AND DISCUSSION}

Three-dimensional boundary-layer flow over a wedge that is considered to be moving either in the same or in the opposite direction to that of the oncoming mainstream flows guides a new family of similarity solutions mainly governed by pressure gradient and shear-to-strain-rate ratio parameter. Three-dimensional boundary-layer flows are typically nonlinear; hence, the asymptotic solutions for $\eta \gg 1$ are obtained based on the linear theory (Section 3) because the velocity profiles away from the wedge surface become linear. However, because of constraints on the arguments of asymptotic velocity profiles $\mathcal{F}(\eta)$ and $\mathcal{G}(\eta)$, the negative $\beta$ results have not been considered. In either of the cases, the solutions are considered with acceleration and deceleration of the mainstream flows where the 3-dimensionality parameter $\alpha$ is also prescribed. In addition, the wall shear stresses $f^{\prime \prime}(0)$ and $g^{\prime \prime}(0)$ and displacement thicknesses $\delta_{x}$ and $\delta_{y}$ are also obtained and presented. These results from both solution methods are further compared to complement each other. Similar to Davey, ${ }^{2}$ we note that the similarity solutions exist only in the range $-1<\alpha<\infty$ and beyond $\alpha \leq-1$, solutions do not exist. To compare the present results with those of existing ones, the skin-friction values 0.774759695 and 1.23259757 are, respectively, obtained for $\beta=0.3$ and $\beta=1.0$ keeping $\lambda_{1}=0=\lambda_{2}$ and $\alpha=0$ constant, which are exactly comparable to the results mentioned by Evans. ${ }^{22}$

Before analysing the constructive nature of the solutions, we first discuss the existence of the eigenvalues $\Delta_{1}$ and $\Delta_{2}$ that decouple the system (7-8). Some of the eigenvalues $\Delta_{1}$ and $\Delta_{2}$ are given in Figure 1 as a function of $\alpha$. This figure clearly shows that the eigenvalues are real and distinct for $\alpha$ and $\beta$ for which the system is decoupled. Note that for $\alpha=-1$ and for all $\beta(\neq 0)$ and for $\beta=0$ and for all $\alpha$, the eigenvalues are equal and zero. The velocity profiles $f^{\prime}(\eta)$ and $g^{\prime}(\eta)$ obtained from (25) exist for $\alpha \in(-1, \infty)$ and for accelerated pressure gradient $\beta(>0)$ because gamma function requires an argument to be positive. Also it is worth mentioning that both eigenvalues $\Delta_{1}$ and $\Delta_{2}$ change their signs exactly at $\alpha=-1$. For $\alpha<-1$ and $\beta \neq 0$, the first argument in $\mathcal{M}$ becomes negative in which case $\mathcal{M} \rightarrow \infty$ and eventually $\mathcal{F}(\eta) \rightarrow \infty$ and $\mathcal{G}(\eta) \rightarrow \infty$ as $\eta \rightarrow \infty$. This violates the derivative conditions at the edge of the boundary layer, $\operatorname{cf}(25)$. Also, $\hat{a}$ becomes negative in (23).

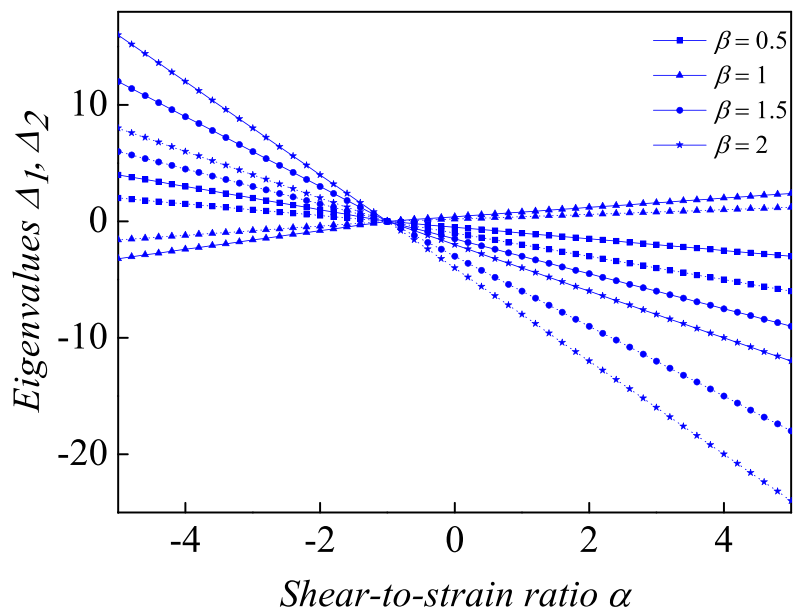

FIGURE 1 Nature of the eigenvalues $\Delta_{1}$ and $\Delta_{2}$ [Colour figure can be viewed at wileyonlinelibrary.com]
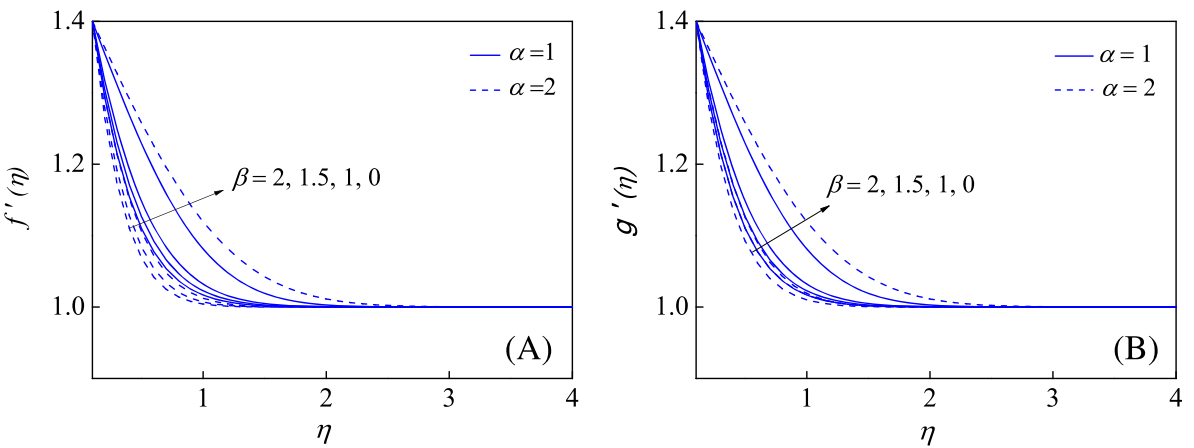

FIGURE 2 Variation of velocity profiles $f^{\prime}(\eta)$ and $g^{\prime}(\eta)$ with $\eta$ for $\beta$ and $\alpha$ [Colour figure can be viewed at wileyonlinelibrary.com] 
In any case, these essentially produce physically irrelevant solutions. The solutions to (7) and (8) exist only for $\alpha>-1$. We, therefore, discuss most of the results for accelerated flows in 3-dimensional boundary layers.

Figure 2 shows the velocity profiles for various values of accelerated pressure gradient and for $\alpha=1$ and $\alpha=2$. The results of $\beta=0$ are obtained from (12) and for other $\beta$ from (25). We see that the velocity profiles do exist for positive values of $\beta$ and are benign in nature. The prominent nature of the accelerated flow is to decrease the horizontal velocity and also to decrease the thickness of the boundary layer. Computations at increasing $\beta$ clearly indicated that the profiles becoming confined to a region close to the moving surface. Thus, for the class of mainstream forcing discussed above, the velocity profiles $f^{\prime}(\eta)$ and $g^{\prime}(\eta)$ exist and found that the flow is always convected towards the wedge surface. Because of moving wedge, the fluid around it pulls towards the surface because viscosity effects lead to thinning of the boundary-layer thickness.

In Figure 3, a similar treatment in which (along with the above results) both wedge and mainstream flows have movements in both directions imposing the same form of pressure gradient $(\beta=2.5)$ and shear-to-strain-rate parameter $\alpha=-0.5$ produced the velocity profiles, which approach the mainstream flows asymptotically. It is observed that the velocity profiles are very different in each direction. In Figure 3A, it is seen that for smaller values of $\lambda_{1}$, the undershoots $\left(f^{\prime}(\eta)<1\right)$ appear near the wedge surface but later merge with outerstream. Nevertheless, Figure 3B shows rather different profiles. For all $\lambda_{2}$, we see that there are overshoots $\left(g^{\prime}(\eta)>1\right)$ near the wedge. Also, boundary-layer domain is quite large compared to $x$-direction domain. The overshoots in $y$-direction seen to appear quite regularly and are a prominent feature of a thickening of the boundary layer.

Along with the results of Figure 3 for $\lambda_{1}$ and $\lambda_{2}$, Figure 4 illustrates how the wall shear stresses vary with $\lambda_{1}$ and $\lambda_{2}$ for 2 sets of $\beta$ and $\alpha$. These results are obtained both numerically (dashed curves) and asymptotically (solid curves). Both wall shear stresses decrease to negative infinity for increasing $\lambda_{1}$ and $\lambda_{2}$. These values for $f^{\prime \prime}(0)$ and $g^{\prime \prime}(0)$ are positive for $\lambda_{1}<1$ and $\lambda_{2}<1$, become zero at $\lambda_{1}=1=\lambda_{2}$, and are negative for $\lambda_{1}>1$ and $\lambda_{2}>1$. At $\lambda_{1}=1=\lambda_{2}$, both wall shear stresses change their trend which is clearly seen from the figure. This typical nature is observed for all $\beta$ and $\alpha$. The corresponding velocity profiles can be realized exactly from Figure 3.
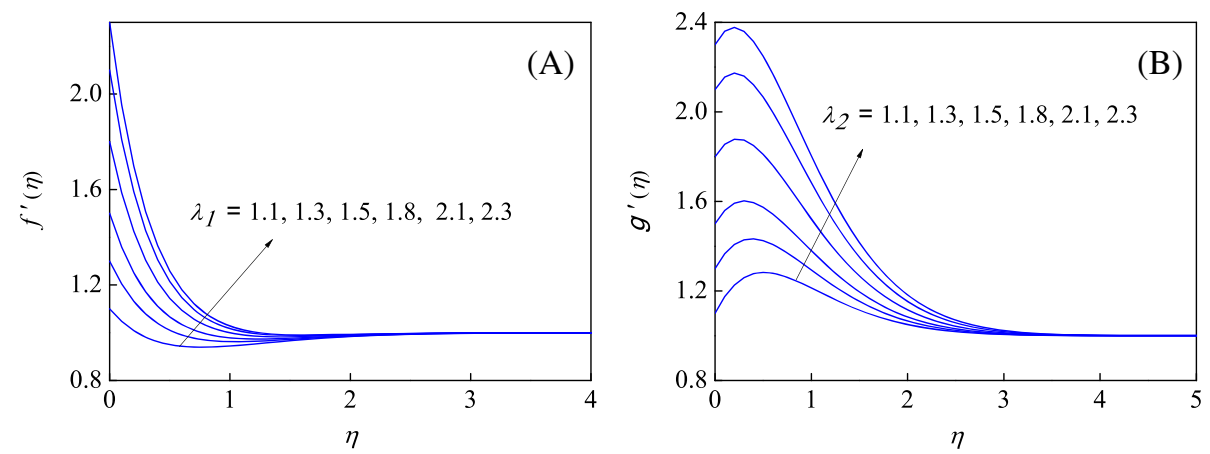

FIGURE 3 Variation of velocity profiles $f^{\prime}(\eta)$ and $g^{\prime}(\eta)$ with $\eta$ for different $\lambda_{1}, \lambda_{2}$, and fixed $\alpha=1.5$ and $\beta=2.5$ [Colour figure can be viewed at wileyonlinelibrary.com]
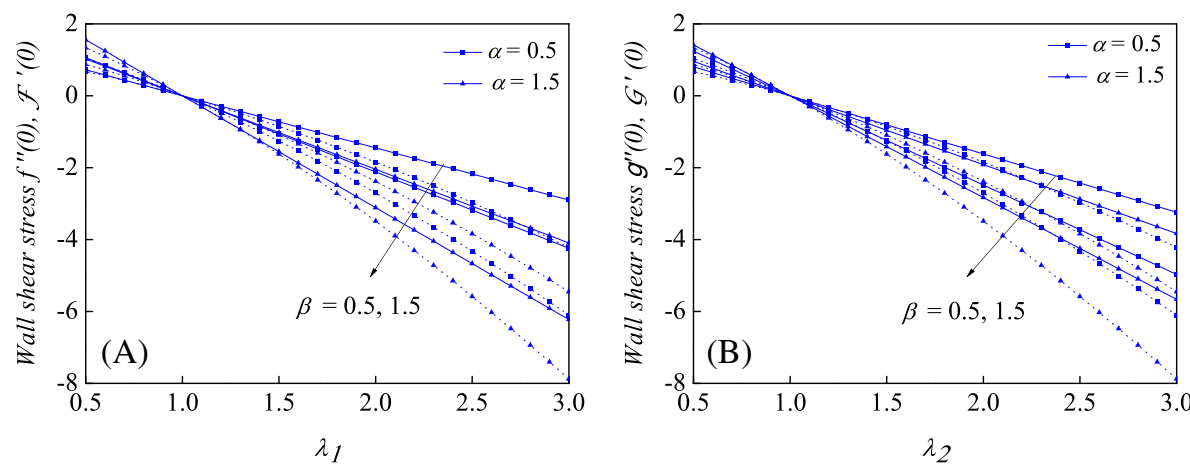

FIGURE 4 Illustration of how the wall shear stresses vary with $\lambda_{1}$ and $\lambda_{2}$ for different $\beta$ and $\alpha$. Here, solid lines are computed from the far-field behavior, and dashed lines are from numerically [Colour figure can be viewed at wileyonlinelibrary.com] 
A number of the flow phenomena considered above (Figures 2 and 3) can be put conveniently in a combination of $\beta$ and $\alpha$ in terms of the wall shear stresses $f^{\prime \prime}(0)$ and $g^{\prime \prime}(0)$. The wall shear stresses $f^{\prime \prime}(0)$ and $g^{\prime \prime}(0)$ are shown in Figure 5 directly computed from the asymptotic solution (solid lines) along with the full numerical solution (dashed lines). Both wall shear stresses grow without bound for increasing $\alpha$ and $\beta$. Similar kind of results are produced in Takhar et al. ${ }^{10}$ In contrast to the above results, very different solutions are shown in Figure 6. The wall shear stress along $x$-direction increases unboundedly, whereas in the $y$-direction $g^{\prime \prime}(0)$ decreases to negative infinity and for some $\alpha$ at which the curves crisscross each other. These surprise results are further confirmed by the full numerical solution of the system. Therefore, to validate these results, we intentionally computed some of the velocity profiles for different values of $\alpha$ in Figure 7 to analyze their nature. In this figure, the observation is that the overshoots $\left(g^{\prime}(\eta)>1.1\right.$ for some $\left.\eta\right)$ appear for smaller values of $\alpha$ (for example $\alpha=0$, the velocity profile attains its overshoot maximum at $\eta=0.33$ and maximum value is
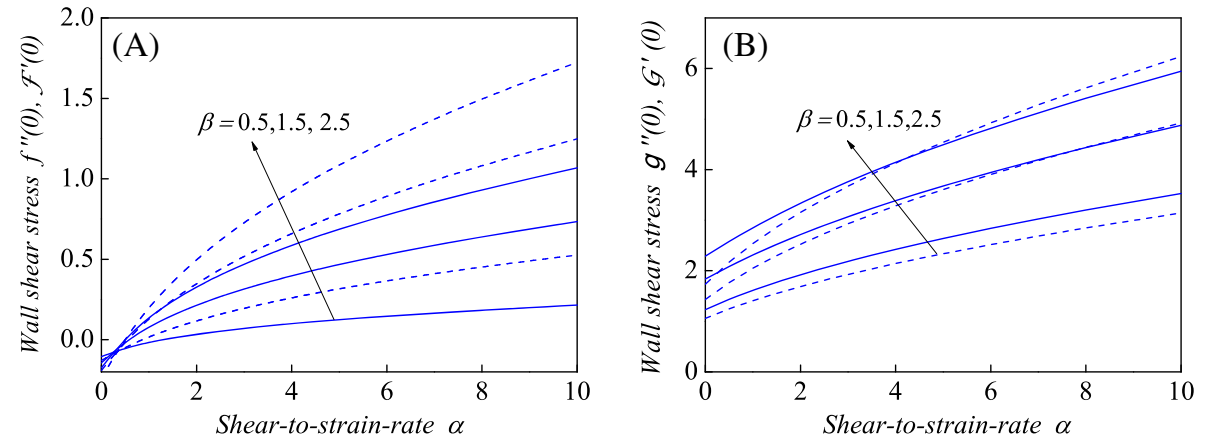

FIGURE 5 Illustration of shear stresses vary with $\alpha$ for different $\beta$ for fixed $\lambda_{1}=1.1$ and $\lambda_{2}=0$. Here, solid lines are computed from the far-field behavior, and dashed lines are from numerically [Colour figure can be viewed at wileyonlinelibrary.com]
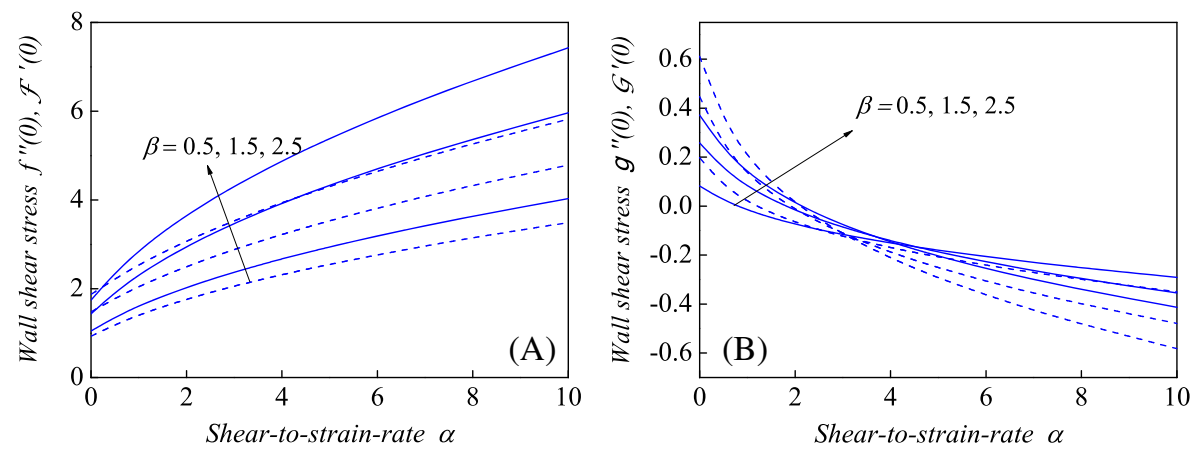

FIGURE 6 Illustration of shear stresses vary with $\alpha$ for different $\beta$ for fixed $\lambda_{1}=0$ and $\lambda_{2}=1.1$. Here, solid lines are computed from the far-field behavior, and dashed lines are from numerically [Colour figure can be viewed at wileyonlinelibrary.com]

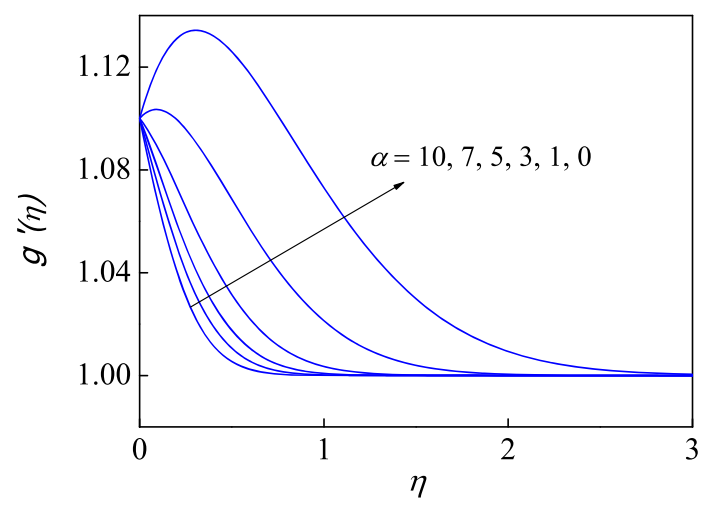

FIGURE 7 Variation of velocity profile $g^{\prime}(\eta)$ with $\eta$ for different values of $\alpha$ and fixed $\beta=1.5$ and $\lambda_{2}=1.1$ and $\lambda_{1}=0$ [Colour figure can be viewed at wileyonlinelibrary.com] 

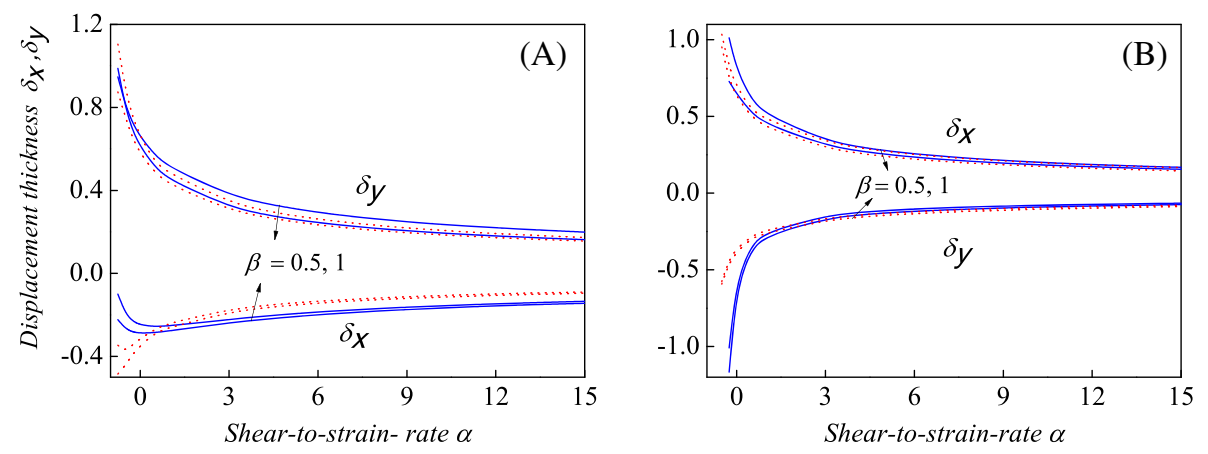

FIGURE 8 Computation of the displacement thicknesses (solid lines) numerically and asymptotically (dashed lines) from (26) for different values of $\alpha$ and $\beta$; (A) $\lambda_{1}=1.1, \lambda_{2}=0$ and (B) $\lambda_{1}=0, \lambda_{2}=1.1$ [Colour figure can be viewed at wileyonlinelibrary.com]

$g^{\prime}(0.33)=1.13411>\lambda_{2}$, similarly $g^{\prime}(0.09)=1.10347>\lambda_{2}$ for $\left.\alpha=1\right)$. The results for $f^{\prime}(\eta)$ are quite benign in nature, hence not shown in the figure. This typical trend is also observed for all favorable pressure gradient parameters.

Asymptotic solutions obviously restrict the class of flows to be considered in 3-dimensional boundary-layer equations, but nonetheless, the resulting class is significant from both practical and theoretical point of view. Arguments in various functions (the gamma or hypergeometric functions) are to be positive. Thus, various physical parameters are so chosen such that arguments are positive. Results further show that the boundary-layer thickness decreases for an accelerated pressure gradient parameter (ie, viscous effects are confined to the moving wedge surface) and divide into near- and far-field regions. In the former region, the flow is dominated by viscous forces, and in the latter case, it is mainly controlled by inviscid mechanisms. Also, the wall shear stresses decrease for increasing mainstream forcing (pressure gradient) that leads fluid to move faster.

Because of various constraints on physical parameters, we solve the full nonlinear system numerically and also to support the results obtained asymptotically (Section 3). In this case, we extensively use the Keller-box solver for the solution. Full numerical solution of the system also confirms the restriction on $\alpha$, ie, solutions do exist only in the range $-1<\alpha<\infty$. This is also reported in the studies of Davey and Schofield. ${ }^{3}$ These numerical solutions always compliment the asymptotic solutions qualitatively, and hence the same results have not been reproduced. Also, in Figures 5 and 6, the wall shear stresses $f^{\prime \prime}(0)$ and $g^{\prime \prime}(0)$ are compared with the asymptotic solutions and found that results are agreeing well. In addition to the above numerical results, Figure 8 discusses the displacement thicknesses computed from (26) (dashed lines, computed from (26) with $\delta_{x 0}=\delta_{x}, \delta_{y 0}=\delta_{y}$ ) and from (34) (solid lines, numerically). Note that there is an excellent agreement between 2 results. Figure 8A indicates that $\delta_{x}\left(\delta_{y}\right)$ increase (decrease) gradually as $\alpha$ increases from -1 . However, Figure $8 \mathrm{~B}$ indicates the opposite results. Weidman ${ }^{4}$ also reported such gradual variations of the displacement thicknesses. The displacement thickness $\delta_{x}\left(\delta_{y}\right)$ decreases (increases) for smaller values of $\alpha$, and after certain $\alpha$, both become parallel (almost) and increase gradually further.

\section{6 | CONCLUSIONS}

In this paper, the numerical and asymptotic analyses are used to investigate the effects of streamwise pressure gradient on 3-dimensional boundary-layer flow of a viscous fluid over a wedge. The partial differential equations governing the 3-dimensional flow have been converted into a system of ordinary differential equations via newly introduced similarity transformations, which have been solved for streamwise velocity profiles and displacement thicknesses. To the best of authors knowledge, linear algebraic approach based asymptotic solutions have not been reported. The results thus obtained are valid for all $\beta$ and $-1<\alpha<\infty$. The various velocity profiles show that the flow divides into near- and far-field region. Further, the thickness of the boundary layer decreases for accelerated pressure gradient and for increasing shear-to-strain-rate parameter.

\section{ACKNOWLEDGEMENTS}

The authors thank Professor A.S. Vasudeva Murthy, TIFRCAM, Bangalore, for useful and constructive discussions. The authors thank all the reviewers for their valuable comments for the improvement of the technical contents. 


\section{ORCID}

\section{Ramesh B. Kudenatti (iD) http://orcid.org/0000-0002-4338-947X}

\section{REFERENCES}

1. Banks WHH. A three-dimensional laminar boundary-layer calculation. J Fluid Mech. 1967;28:769-792.

2. Davey A. Boundary layer flow at a saddle point of attachment. J Fluid Mech. 1961;10:593-610.

3. Davey A, Schofield D. Three-dimensional flow near a two -dimensional stagnation point. J Fluid Mech. 1967;28:149-151.

4. Weidman PD. Non-axisymmetric Homann stagnation-point flows. J Fluid Mech. 2012;702:460-469.

5. Kudenatti RB, Kirsur SR. Numerical and asymptotic study of non-axisymmetric magnetohydrodynamic boundary layer stagnation-point flows. Math Methods Appl Sci. 2017;40:5841-5850.

6. Khan JA, Mustafa M, Hayat T, Alsaedi A. On three-dimensional flow and heat transfer over a non-linearly stretching sheet: analytical and numerical solutions. Plos One. 2014;9(9):e107287.

7. Turkyilmazoglu M. Equivalences and correspondences between the deforming body induced flow and heat in two-three dimensions. Phys Fluids. 2016;28:043102-1-10.

8. Turkyilmazoglu M. Stretching/shrinking longitudinal fins of rectangular profile and heat transfer. Energy Convers Manag. 2015;91:199-203.

9. Wang CY. Three-dimensional flow due to stretching flat surface. Phys Fluids. 1984;27:1915-1917.

10. Takhar HS, Chamkha AJ, Nath G. Unsteady three dimensional MHD boundary layer flow due to the impulsive motion of a stretching surface. Acta Mech. 2001;146:59-71.

11. Tzirtzilakis EE, Kafoussias NG. Three-dimensional magnetic fluid boundary-layer flow over a linearly stretching sheet. ASME: $J$ Heat Transfer. 2010;132:011702-1-8.

12. Dhanak MR, Duck PW. The effects of freestream pressure gradient on a corner boundary layer. Proc R Soc A. 1997;453:1793-1815.

13. Liu I-C, Wang H-H, Peng Y-F. Flow and heat transfer for three-dimensional flow over an exponentially stretching surface. Chem Eng Commun. 2014;200:253-268.

14. Hayat T, Aziz A, Muhammad T, Alsaedi A. On magnetohydrodynamic three-dimensional flow of nanofluid over a convectively heated nonlinear stretching surface. Int J Heat Mass Transfer. 2016;100:566-572.

15. Muhammad T, Alsaedi A, Hayat T, Shehzad SA. A revised model for Darcy-Forchheimer three-dimensional flow of nanofluid subject to convective boundary condition. Results Phys. 2017;7:2791-2797.

16. Schlichting H, Gersten K. Boundary Layer Theory. 8th ed. New York: Springer; 2004.

17. Kudenatti RB, Kirsur SR, Achala LN, Bujurke NM. MHD boundary layer flow over a non-linear stretching boundary with suction and injection. Int J Nonlinear Mech. 2013;50:58-67.

18. Abramowitz M, Stegun I. Handbook of Mathematical Functions with Formulas, Graph and Mathematical Tables. 9th ed. New York: Dover publications; 1970.

19. Andrews L. Special Functions of Mathematics for Engineers. 2nd ed. Oxford, UK: Oxford University Press; 1998.

20. Cebeci T, Bradshaw P. Momentum Transfer in Boundary Layers. New York: Mc. Graw Hill; 1977.

21. Keller HB. Numerical methods in boundary layer theory. Annu Rev Fluid Mech. 1978;10:417-428.

22. Evans HL. Laminar Boundary-Layer Theory. United States: Addison-Wesley publishing company; 1968.

How to cite this article: Kudenatti RB, Gogate SSP, Bujurke NM. Asymptotic and numerical solutions of three-dimensional boundary-layer flow past a moving wedge. Math Meth Appl Sci. 2018;41:2602-2614. https://doi.org/10.1002/mma.4761 